Research Article

\title{
The Beckman-Quarles Theorem in Hyperbolic Geometry
}

\author{
Oğuzhan Demirel $(\mathbb{D}$, Damla Topal $\mathbb{D}$, and Leyla Aslan \\ Afyon Kocatepe University, Department of Mathematics, Faculty of Science and Literature, Ahmet Necdet SEZER Campus, \\ Afyon 03200, Turkey
}

Correspondence should be addressed to Oğuzhan Demirel; odemirel@aku.edu.tr

Received 6 January 2021; Revised 19 February 2021; Accepted 25 February 2021; Published 16 March 2021

Academic Editor: Antonio Masiello

Copyright (c) 2021 Oğuzhan Demirel et al. This is an open access article distributed under the Creative Commons Attribution License, which permits unrestricted use, distribution, and reproduction in any medium, provided the original work is properly cited.

In this paper, we present the counterpart of the Beckman-Quarles theorem in the Poincare disc model of hyperbolic geometry to characterize the gyroisometries (hyperbolic isometries) with a single nonzero distance $a \in(0,1)$ satisfying $a^{2} \in \mathbb{Q}$.

\section{Introduction}

Let $(X, d)$ be a metric space and $f$ be a function defined from $(X, d)$ to itself. If $d(f(P), f(Q))=d(P, Q)$ holds for all $P, Q \in X$, then $f$ is called an isometry of $X$. An isometry is injective, but it need not be surjective. If an isometry is invertible, its inverse is also an isometry. An isometry of Euclidean space $\mathbb{R}^{n}$ is bijective. The set Iso $\left(\mathbb{R}^{n}\right)$ of all isometries of $\mathbb{R}^{n}$ is a group under composition. It is well known that every isometry of $\mathbb{R}^{n}$ is a composition of at most $n+1$ reflections. An isometry that fixes at least one point is a composition of at most $n$ reflections.

Let us consider the Euclidean space $\mathbb{R}^{n}(n \geq 2)$ and $f$ be a function defined from $\mathbb{R}^{n}$ to itself. The Beckman-Quarles [1] theorem states that if $f$ is a function defined from $\mathbb{R}^{n}(n \geq 2)$ to itself which preserves a distance $k \in \mathbb{R}^{+}$, then it is an isometry of $\mathbb{R}^{n}$. In the literature, there are several proofs of this famous theorem, see [2, 3], and some authors [4-7] tried to find the counterpart of this theorem in various spaces.

Hyperbolic geometry is a non-Euclidean geometry that rejects the validity of Euclid's fifth postulate. The principles of hyperbolic geometry, however, admit the other four Euclidean postulates. Although many of the theorems of hyperbolic geometry are identical to those of Euclidean, others differ. For example, in Euclidean geometry, two parallel lines are taken to be everywhere equidistant. In hyperbolic geometry, two parallel lines are taken to converge in one direction and diverge in the other. In Euclidean geometry, the sum of the angles in a triangle is equal to two right angles; in hyperbolic geometry, the sum is less than two right angles. In Euclidean geometry, polygons of differing areas can be similar, and in hyperbolic geometry, similar polygons of differing areas do not exist. There are many principal hyperbolic geometry models, for instance, Weierstrass model, Beltrami-Klein model, Poincaré disc model, and Poincaré upper-half plane model. In this paper, we deal with the Poincaré disc model of hyperbolic geometry to get the desired results. The points of this model are the points of the complex unit disc:

$$
\mathbb{D}=\{z \in \mathbb{C}:|z|<1\},
$$

and the hyperbolic lines are circular arcs orthogonal to the boundary circle of the disc including the diameters of $\mathbb{D}$. Two arcs which do not meet correspond to parallel rays and the arcs which meet orthogonally correspond to perpendicular lines (see Figure 1). The hyperbolic angle between two hyperbolic lines is the usual Euclidean angle between Euclidean tangents to the circular arcs. The advantage of the Poincare disc model is that it is conformal, namely, circles and angles are not distorted.

The classical hyperbolic distance $d_{H}$ in $\mathbb{D}$ is defined by

$$
d_{H}\left(z_{1}, z_{2}\right)=\frac{1}{2} \ln \frac{1+d\left(z_{1}, z_{2}\right)}{1-d\left(z_{1}, z_{2}\right)},
$$

where 


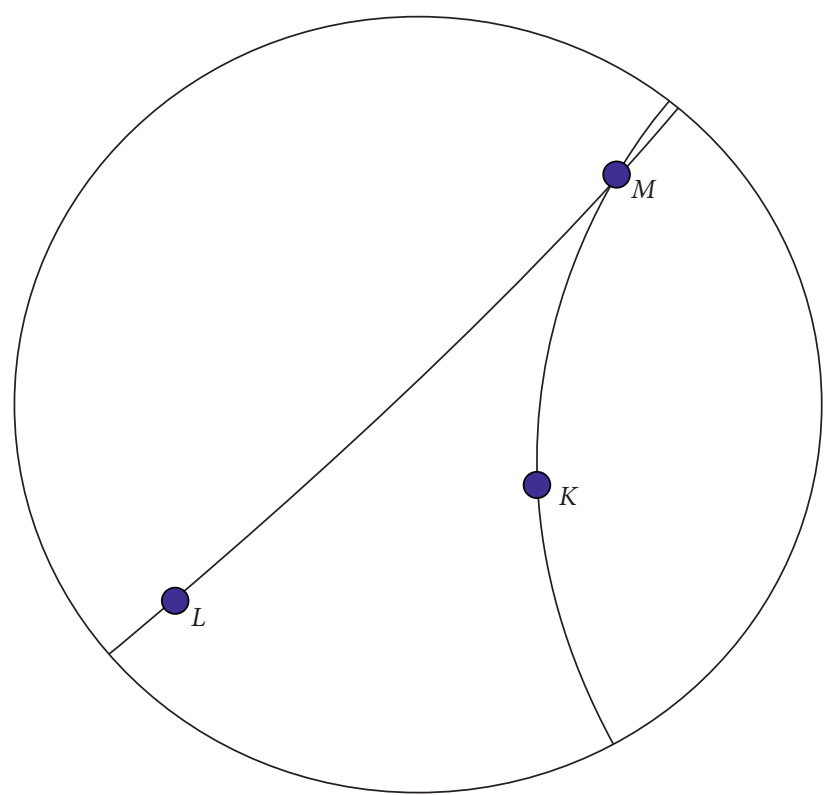

Figure 1: A gyroline (hyperbolic line) passing through the points $M$ and $K$ is a circular arc that meets the boundary of the disc $\mathbb{D}$ orthogonally. The hyperbolic lines passing through the center of disc are also corresponding to chords of the disc.

$$
d\left(z_{1}, z_{2}\right)=\left|\frac{z_{1}-z_{2}}{1-\overline{z_{1}} z_{2}}\right|,
$$

for all $z_{1}, z_{2} \in \mathbb{D}$.

A Möbius transformation $f$ of the extended complex plane $\mathbb{C} \cup\{\infty\}$ is a rational function of the form

$$
f(z)=\frac{a z+b}{c z+d}
$$

of one complex variable $z$; here, the coefficients $a, b, c, d$ are complex numbers satisfying $a d-b c \neq 0$. Möbius transformations are named in honor of August Ferdinand Möbius; they are also variously named bilinear transformations, homographic transformations, or fractional linear transformations. The set of all Möbius transformations forms a group under composition. Möbius transformations preserve the measures of the angles with orientation. Euclidean transformations, Euclidean rotations, inversions, and similarities are well-known Möbius transformations.

The most general Möbius transformation of the complex unit disc $\mathbb{D}$ in the complex plane to itself

$$
z \mapsto e^{i \theta} \frac{z_{0}+z}{1+\overline{z_{0}} z}=e^{i \theta}\left(z_{0} \oplus z\right)
$$

defines the Möbius addition in the disc, which allows the Möbius transformation of the disc to be viewed as a Möbius left translation $[8,9]$ :

$$
z \mapsto z_{0} \oplus z=\frac{z_{0}+z}{1+\overline{z_{0}} z}
$$

where $\theta \in \mathbb{R}, z_{0} \in \mathbb{D}$, and $\overline{z_{0}}$ is the complex conjugate of $z_{0}$. A left Möbius translation is also called a left gyrotranslation [8]. It is known that the Möbius addition " $\oplus$ " is analogous to the common vector addition " + " in Euclidean plane geometry. Möbius addition $\oplus$ is neither commutative nor associative. By defining the gyrator

$$
\begin{aligned}
& \text { gyr: } \mathbb{D} \times \mathbb{D} \longrightarrow \operatorname{Aut}(\mathbb{D}, \oplus), \\
& \operatorname{gyr}[a, b]=\frac{a \oplus b}{b \oplus a}=\frac{1+a \bar{b}}{1+b \bar{a}},
\end{aligned}
$$

where $\operatorname{Aut}(\mathbb{D}, \oplus)$ is the automorphism group of the Möbius groupoid $(\mathbb{D}, \oplus)$, the following group-like properties of $\mathbb{D}$ can be verified by straightforward algebra for all $a, b, c \in \mathbb{D}$ :

G1. $a \oplus b=\operatorname{gyr}[a, b](b \oplus a)$ (gyrocommutative law)

G2. $a \oplus(b \oplus c)=(a \oplus b) \operatorname{gyr}[a, b] c$ (left gyroassociative law)

G3. $(a \oplus b) \oplus c=a \oplus b(\operatorname{gyr}[b, a] c)$ (right gyroassociative law)

G4. $\operatorname{gyr}[a, b]=\operatorname{gyr}[a \oplus b, b]$ (left loop property)

G5. $\operatorname{gyr}[a, b]=\operatorname{gyr}[a, b \oplus a]$ (right loop property)

G6. $a \oplus b=\operatorname{gyr}[a, b](b \oplus a)$ (gyrocommutative law)

The Möbius gyrodistance function $d_{M}$ in $\mathbb{D}$ is

$$
d_{M}(z, w)=|z \ominus w|=\left|\frac{z-w}{1-\bar{z} w}\right|
$$

which is closely related to classical hyperbolic distance $d_{H}$ as follows:

$$
\tanh d_{H}(z, w)=\left|\frac{z-w}{1-\bar{z} w}\right|=d_{M}(z, w)
$$

The operations “ $\oplus$ " and “ $\ominus$ " are called Möbius addition and Möbius subtraction, respectively. $d_{M}$ is not a metric on $\mathbb{D}$ since the triangle inequality is not provided. The gyrodistances are invariant under left gyrotranslations and rotations about origin. Clearly, $d_{H}\left(z_{1}, z_{2}\right) \in[0, \infty)$ and 
$d_{M}\left(z_{1}, z_{2}\right) \in[0,1)$ for all $z_{1}, z_{2} \in \mathbb{D}$. The prefix "gyro" stems from Thomas gyration which is, in turn, the mathematical abstraction of a special relativistic effect known as Thomas precession $[8,9]$. In gyrolanguage, we prefix a gyro to any term describes a concept in Euclidean geometry and in associative algebra to mean the analogous concept in hyperbolic geometry and nonassociative algebra. In full analogy with Euclidean geometry, a gyrotriangle consists of three line segments called sides or edges and three points called vertices (see Figure 2). The following theorems about gyrotriangles play major roles in our results.

Theorem 1. Let $A B C$ be a gyrotriangle in $\mathbb{D}$ with vertices $A, B, C$ corresponding gyroangles $\alpha, \beta, \gamma$ and side gyrolengthsa, $b, c$. The sides of the gyrotriangle $A B C$ are determined by its gyroangles by

$$
\begin{aligned}
& a^{2}=\frac{\cos \alpha+\cos (\beta+\gamma)}{\cos \alpha+\cos (\beta-\gamma)}, \\
& b^{2}=\frac{\cos \beta+\cos (\alpha+\gamma)}{\cos \beta+\cos (\alpha-\gamma)}, \\
& c^{2}=\frac{\cos \gamma+\cos (\alpha+\beta)}{\cos \gamma+\cos (\alpha-\beta)} .
\end{aligned}
$$

Theorem 1 presents a most important disanalogy with Euclidean triangle similarity. For the proof of Theorems 1 and 2, we refer the reader to [9].

Theorem 2. Let $A B C$ be an equilateral gyrotriangle in $\mathbb{D}$ with vertices $A, B, C$ satisfying $\angle A B C=\angle B C A=\angle C A B=\alpha$, the side gyrolengths of which are $a$, and then $a=\sqrt{2 \cos \alpha-1}$.

\section{The Beckman-Quarles Theorem in Hyperbolic Geometry}

Throughout the paper, we denote by $X^{\prime}$ the image of $X$ under $f$, by $[P, Q]$ the gyrosegment that links the points $P$ and $Q$, by $P Q$ the gyroline through the points $P$ and $Q$, by $P Q R$ the gyrotriangle with three ordered vertices $P, Q, R$, by $d(P, Q)$ the Möbius gyrodistance between $P$ and $Q$, by $P Q R S$ the gyroquadrilateral with four ordered vertices $P, Q, R, S$, and by $\angle P Q R$ the gyroangle between $[P, Q]$ and $[R, Q]$. We say that $f$ preserves $s$ if any points $A, B \in \mathbb{D}$ such that $d(A, B)=$ salso satisfy $d(f(A), f(B))=s$.

Lemma 1. If $f: \mathbb{D} \longrightarrow \mathbb{D}$ preserves some gyrodistance $a \in(0,1)$, then it is single valued.

Proof. Assume that $f$ be a nonsingle valued and $A$ be a point in $\mathbb{D}$ such that $f(A)=U, f(A)=V, U \neq V$. Now, construct an equilateral gyrotriangle $A B C$ satisfying $d(A, B)=d(B, C)=d(A, C)=a$. Then, hypothesis $A^{\prime} B^{\prime} C^{\prime}$ is also an equilateral hyperbolic triangle with $d\left(A^{\prime}, B^{\prime}\right)=d\left(A^{\prime}, C^{\prime}\right)=d\left(B^{\prime}, C^{\prime}\right)=a$. Assume $\angle A B C=\alpha$. Then, we get $a^{2}=2 \cos \alpha-1$ which implies $\cos \alpha=\left(a^{2}+\right.$

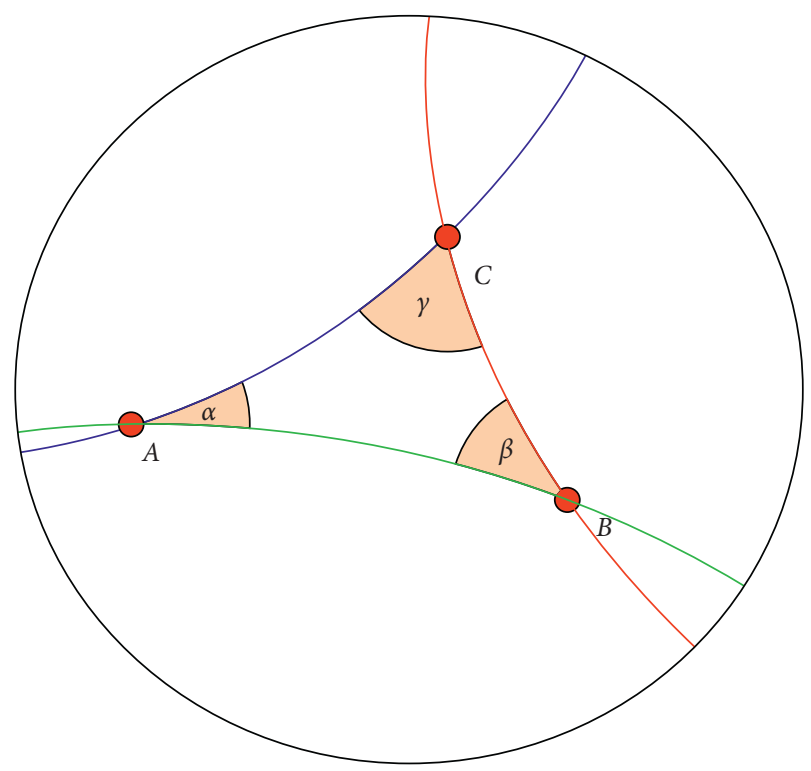

Figure 2: A gyrotriangle (hyperbolic triangle) in the complex unit disc $\mathbb{D}$.

1)/2 by Theorem 2. Now, construct the gyrorhombus $A B D C$ with the help of the gyrotriangle $A B C$. Hence, by Theorem 1, we get

$$
d(A, D)=\sqrt{\frac{\cos 2 \alpha+\cos \alpha}{\cos 2 \alpha+1}}=\sqrt{1+\frac{a^{2}-1}{\left(a^{2}+1\right)^{2}}} .
$$

Since there are no points other than $U$ and $V$ at distance $a$ from $B^{\prime}$ to $C^{\prime}$, one can easily get $D^{\prime}=U$ or $D^{\prime}=V$. Let $g_{\theta}$ be the hyperbolic rotation with respect to $A$ for an appropriate $\theta \in \mathbb{R}$ satisfying $d\left(D, g_{\theta}(D)\right)=a$ and $\angle g_{\theta}(D) A D=\theta$. Clearly, $g_{\theta}(x)=A \oplus\left(e^{i \theta}(\ominus A \oplus X)\right),(\ominus A=-A)$ for $X \in \mathbb{D}$. Let us denote $g_{\theta}(D)=E$. Clearly, $d(A, D)=d(A, E)$ holds and the gyroquadrilateral $A g_{\theta}(B) E g_{\theta}(C)$ is a gyrorhombus congruent to $A B C D$. Since $d\left(g_{\theta}(B), A\right)=a, d$ $\left(g_{\theta}(C), A\right)=a$, we get $d\left(f\left(g_{\theta}(B)\right), U\right)=d\left(f\left(g_{\theta}(B)\right), V\right)=$ $a$ and $d\left(f\left(g_{\theta}(C)\right), U\right)=d\left(f\left(g_{\theta}(C)\right), V\right)=a$. This yields $\left\{f\left(g_{\theta}(B)\right), f\left(g_{\theta}(C)\right)\right\}=\left\{B^{\prime}, C^{\prime}\right\}$, and this implies $E^{\prime}=U$ or $E^{\prime}=V$. Therefore, we get $d\left(D^{\prime}, E^{\prime}\right) \epsilon$ $\left\{0, \sqrt{1+\left(\left(a^{2}-1\right) /\left(a^{2}+1\right)^{2}\right)}\right\}$, but since $d(D, E)=a$, this is a contradiction.

Corollary 1. If $f: \mathbb{D} \longrightarrow \mathbb{D}$ is a map which preserves some gyrodistance $a \in(0,1)$, then it preserves the gyrodistance $\sqrt{1+\left(\left(a^{2}-1\right) /\left(a^{2}+1\right)^{2}\right)}$.

Proof. Let us consider the gyrorhombuses $A B D C$ and $A g_{\theta}(B) E g_{\theta}(C)$ in Lemma 1. Clearly, $d(A, D)=d(A, E)=$ $\sqrt{1+\left(\left(a^{2}-1\right) /\left(a^{2}+1\right)^{2}\right)}$ and this implies $d\left(A^{\prime}, D^{\prime}\right)=0$ or $\left(A^{\prime}, D^{\prime}\right)=\sqrt{1+\left(\left(a^{2}-1\right) /\left(a^{2}+1\right)^{2}\right)}$. Assume $d\left(A^{\prime}, D^{\prime}\right)=0$. Hence, we get $A^{\prime}=D^{\prime}$ and this implies $d\left(A^{\prime}, E^{\prime}\right)=$ 
$d\left(D^{\prime}, E^{\prime}\right)=0 \quad$ or $\quad d\left(A^{\prime}, E^{\prime}\right)=d \quad\left(D^{\prime}, E^{\prime}\right)=$ $\sqrt{1+\left(\left(a^{2}-1\right) /\left(a^{2}+1\right)^{2}\right)}$. Since $d(D, E)=a$ holds, this is a contradiction. Thus, we get $\left(A^{\prime}, D^{\prime}\right)=$ $\sqrt{1+\left(\left(a^{2}-1\right) /\left(a^{2}+1\right)^{2}\right)}$.

Let us define $\psi(a)=\sqrt{1+\left(\left(a^{2}-1\right) /\left(\left(a^{2}+1\right)^{2}\right)\right)}$ and $\psi^{n}(a)=\psi(\psi(\ldots, \psi(a), \ldots)) \quad(n-$ times $)$ for all $n \in \mathbb{N}$. Clearly, $a<\psi(a)$ and $\psi^{i}(a)<\psi^{j}(a)$ hold if $i<j$.

Corollary 2. If $f: \mathbb{D} \longrightarrow \mathbb{D}$ is a map which preserves some gyrodistance $a \in(0,1)$, then it preserves the gyrodistances $\psi^{n}(a)$ for all $n \in \mathbb{N}$.

Theorem 3. If $f: \mathbb{D} \longrightarrow \mathbb{D}$ is a map which preserves some gyrodistance $a \in(0,1)$ satisfying $a^{2} \in \mathbb{Q}$, then $f$ preserves all gyrodistances.

\section{Proof}

Step 1. We claim that $f$ preserves the gyrodistances less than $a$. Let $P$ and $Q$ be two distinct points in $\mathbb{D}$ satisfying $d(P, Q)<a$. Clearly, there exist two points in $\mathbb{D}$, say $C$ and $E$, such that $d(P, C)=a=d(E, C)$ and $Q \in[C, E]$. Let $S(C, a)$ be the gyrocircle centered at $C$ with radius $a$. Then, there exist two points on $S(C, a)$, say $R_{1}$ and $R_{2}$ such that $d\left(P, R_{1}\right)=d\left(P, R_{2}\right)=a$. Thus, we construct two equilateral gyrotriangles $P R_{1} C, P R_{2} C$, the side gyrolengths of which are $a$. Here, the point $Q$ can be thought to be on the gyrosegment $\left[P, R_{1}\right]$, otherwise a new configuration is needed to provide this feature, which is not difficult to construct. Assume $\angle P R_{1} C=\angle P R_{2} C=\alpha$. Now, construct the sequence $A_{i} C A_{i+1}(i=1,2, \ldots)$ with $d\left(C, A_{i}\right)=d\left(C, A_{i+1}\right)=d\left(A_{i}, A_{i+1}\right)=a, A_{1}=P, A_{2}=R_{1}$, $\angle A_{i} C A_{i+1}=\alpha$. It is clear that there is another sequence $B_{i} C B_{i+1}(i=1,2, \ldots)$ with $d\left(C, B_{i}\right)=d\left(C, B_{i+1}\right)=d\left(B_{i}, B_{i+1}\right)=$ a, $B_{1}=P, B_{2}=R_{2}, \angle B_{i} C B_{i+1}=\alpha$. If it can be established that $\alpha \notin \pi \mathbb{Q}$, then there is no nonzero integer $k$ such that $A_{1}=A_{k}$. If there is such a $k$ satisfying $A_{1}=A_{k}$, then one can easily see that $k \alpha=2 \pi$, i.e., $\alpha=(2 \pi / k) \in \pi \mathbb{Q}$. In [3], it was shown that if $\rho=(m / n) \pi$, where $m, n \in \mathbb{Z}$ satisfying $\cos \rho \in \mathbb{Q}, \quad$ then $\cos \rho \in\{0, \pm(1 / 2), \pm 1\}$. Since $\cos \alpha=\left(\left(a^{2}+1\right) / 2\right) \notin\{0, \mp(1 / 2), \mp 1\}$ for all $a \in(0,1)$ satisfying $a^{2} \in \mathbb{Q}$, we immediately get that $\alpha \notin \pi \mathbb{Q}$. Hence, the gyrotriangles which define the sequence $A_{i} C A_{i+1}$ touch the gyroarc $\operatorname{arc}\left(\mathrm{PR}_{1}\right)$ at an infinite number of points. Thus, $\left[P, R_{1}\right]$ has been divided into gyrosegments as desired, and the images of the points that provide this disintegration are gyrocollinear. Moreover, the gyrodistances between the points that provide this disintegration are also preserved. Thus, $f$ preserves the gyrodistances less than $a$. Hence, we obtain that $d(P, Q)=d\left(P^{\prime}, Q^{\prime}\right)$ holds.

Step 2. We claim that $f$ preserves the gyrodistance $2 \otimes a=a \oplus a$. Let $K$ and $L$ be two distinct points in the gyrocircle $S(C, a)$ satisfying $d(K, L)=2 \otimes a$. Since $f$ preserves the measure of the equilateral gyrotriangles with their gyroangle $\alpha$, constructing a similar sequence in Step 1 above, we immediately get $d\left(K^{\prime}, L^{\prime}\right)=2 \otimes a$. Similarly, it is clear that $f$ also preserves the gyrodistances $n \otimes a=$ $a \oplus \cdots \oplus a$ ( $n$-times) for all $n \in \mathbb{N}$. Notice that since $a \in(0,1)$ and $a^{2} \in \mathbb{Q}$, this implies $n \otimes a \in(0,1)$ and $(n \otimes a)^{2} \in \mathbb{Q}$. Moreover, for each equilateral gyrotriangle $A B C$ with $d(A, B)=d(A, C)=d(B, C)=n \otimes a$ $\left(a^{2} \in \mathbb{Q}\right)$, if $\angle A B C:=\beta$, then $\cos \beta \in \mathbb{Q}$ and $\beta \notin \pi \mathbb{Q}$.

Step 3. $f$ preserves the gyrodistances greater than $a$. Let $P$ and $Q$ be two distinct points in $\mathbb{D}$ satisfying $d(P, Q)>a$. Let $k$ be a positive integer such that $d(P, Q)<k \otimes a$. As in Step 1 , one can easily construct a gyrocircle with radius $k \otimes a$ passing through $P$. Denote this gyrocircle by $(D, k \otimes a)$ where $D$ is its center. Then, there exists a point $W$ on $S(D, k \otimes a)$ such that $D P W$ is an equilateral gyrotriangle and denote $\angle D P W=\phi$. Since $\cos \phi \in \mathbb{Q}$ by Step 2, $f$ preserves all the gyrodistances less than $k \otimes a$. Hence, we see that $f$ preserves all gyrodistances.

\section{Conclusions}

We have proved that if $f: \mathbb{D} \longrightarrow \mathbb{D}$ is a mapping which preserves some gyrodistance $a \in(0,1)$ satisfying $a^{2} \in \mathbb{Q}$, then $f$ is a gyroisometry; that is, it preserves all gyrodistances. This implies that $f$ is also a hyperbolic isometry. Naturally, one may wonder whether Theorem 3 is valid for an arbitrary gyrodistance $a$ satisfying $a^{2} \in(0,1) \backslash \mathbb{Q}$. We leave the solution of this problem to the reader's attention.

\section{Data Availability}

No data were used to support this study.

\section{Conflicts of Interest}

The authors declare that they have no conflicts of interest.

\section{References}

[1] F. S. Beckman and D. A. Quarles, "On isometries of Euclidean spaces," Proceedings of the American Mathematical Society, vol. 4 , no. 5, p. 810, 1953.

[2] W. Benz, "An elementary proof of the theorem of Beckman and Quarles," Elemente der Mathematik, vol. 42, no. 1, pp. 4-9, 1987.

[3] https://mysite.science.uottawa.ca/hsalmasi/report/report-gail. pdf.

[4] J. Zaks, "A discrete form of the Beckman-Quarles Theorem for rational spaces," Journal of Geometry, vol. 72, no. 1-2, pp. 199-205, 2001.

[5] J. Zaks, "The Beckman-Quarles Theorem for rational spaces," Discrete Mathematics, vol. 265, no. 1-3, pp. 311-320, 2003.

[6] A. Tyzska, "Discrete version of the Beckman-Quarles theorem," Aequationes Mathematicae, vol. 59, no. 1-2, pp. 124-133, 2000.

[7] A. Tyzska, "A discrete form of the Beckman-Quarles theorem for rational eight-space," Aequationes Mathematicae, vol. 1-2, pp. 85-93, 2001.

[8] A. A. Ungar, Analytic Hyperbolic Geometry and Albert Einstein's Special Theory of Relativity, World Scientific Publishing Co. Pte. Ltd., Hackensack, NJ, USA, 2008.

[9] A. A. Ungar, Analytic Hyperbolic Geometry: Mathematical Foundations and Applications, World Scientific, Singapore, 2005. 